\title{
Effects of CASZI on bronchopulmonary development of neonatal rats
}

\author{
LIPING XU ${ }^{1 *}$, RUIFANG TONG ${ }^{1 *}$, XIANGNAN LI ${ }^{2}$, LIHONG LIANG $^{1}$ and HAN HUANG ${ }^{1}$ \\ ${ }^{1}$ Department of Respiratory Medicine, Children's Hospital of Zhengzhou, Zhengzhou, Henan 450000; \\ ${ }^{2}$ Department of Respiratory Medicine, Henan Provincial People's Hospital, Zhengzhou, Henan 450003, P.R. China
}

Received June 1, 2017; Accepted October 4, 2017

DOI: $10.3892 /$ etm.2017.5346

\begin{abstract}
The effect of CASZI on bronchopulmonary development in neonatal rats was investigated. Forty SD rats were randomly divided into the control group $(n=20)$ and the experimental group $(n=20)$ using a random number table. The experimental group was treated with hyperoxia to establish the bronchopulmonary dysplasia (BPD) model, while the control group received no treatment. At 14 days of experiment, rats in each group were treated, and lung tissues were taken for hematoxylin and eosin (H\&E) staining. Radial alveolar count (RAC) and morphological changes in lung tissues were observed under a microscope, and the thickness of respiratory membrane was detected via IPP6 image. Reverse transcriptionpolymerase chain reaction (RT-PCR) and western blotting were used to detect the expression levels of CASZI, Rho kinase, RhoA and ET-A receptor in lung tissues. There were no obviously pathological changes in lung tissues of neonatal rats in the control group, while BPD could be seen in lung tissue structures of neonatal rats in the experimental group. RAC in experimental group was significantly lower than that in the control group $(\mathrm{P}<0.05)$, and the thickness of respiratory membrane was significantly higher than that in the control group $(\mathrm{P}<0.05)$. CASZI mRNA expression level in the experimental group was significantly lower than that in control group $(\mathrm{P}<0.05)$, and Rho kinase, RhoA and ET-A receptor mRNA expression levels were significantly higher than those in the control group $(\mathrm{P}<0.05)$. CASZI protein expression level in the experimental group was significantly lower than that in the control group $(\mathrm{P}<0.05)$, and the Rho kinase, RhoA and ET-A receptor protein expression levels were significantly higher than those in the control group $(\mathrm{P}<0.05)$. In conclusion, CASZI expression was downregulated during the development of BPD. CASZI downregulation may have an effect on the pulmonary
\end{abstract}

Correspondence to: Dr Han Huang, Department of Respiratory Medicine, Children's Hospital of Zhengzhou, 255 Gangdu Street, Jinshui, Zhengzhou, Henan 450000, P.R. China

E-mail:hphbtn@163.com

*Contributed equally

Key words: bronchopulmonary dysplasia, CASZI, RhoA/ROCK microvascular regeneration through RhoA/ROCK signaling pathway, thus participating in the pathogenesis of BPD.

\section{Introduction}

Bronchopulmonary dysplasia (BPD) is a common chronic lung disease in neonates, and an important cause of neonatal death. BPD is closely related to infection, premature delivery and long-term inhalation of high-concentration oxygen, characterized by alveolar developmental block and impaired pulmonary microvascular development (1). This disease has a high mortality rate, and survivors are often suffering from lung dysfunction and lung developmental disorder, even delayed neurodevelopment and cerebral palsy (2). In recent years, with the continuous development of medical technology, the incidence rate of typical BPD has been gradually reduced, but that of new BPD remained unchanged (3). Previous studies have confirmed that pulmonary angiogenesis is the prerequisite for normal alveolar development. This process involves mutual regulation among multiple factors, among which the transcription factor CASZI is an important factor in pulmonary angiogenesis $(4,5)$. Only few studies have been conducted on the role of CASZI in bronchopulmonary development, and in this study we analyzed the relationship between CASZI and pulmonary angiogenesis using neonatal rats exposed to hyperoxia.

\section{Materials and methods}

Experimental animals and groups. Forty Sprague-Dawley neonatal rats aged 1-3 days of either sex were purchased from Shanghai Experimental Animal Center. Rats were randomly divided into the control group $(\mathrm{n}=20)$ and the experimental group $(n=20)$ using a random number table.

Materials. We used total RNA extraction kit; RT-PCR kit (both from Active Motif, Carlsbad, CA, USA); rabbit-anti mouse $\beta$-actin monoclonal antibody (dilution, 1:1,000; cat. no. CW0096; Beijing ComWin Biotech Co., Ltd., Beijing, China); whole protein extraction kit (Beyotime Biotechnology Institute); rabbit-anti mouse CASZ1, Rho kinase, RhoA and ET-A receptor monoclonal antibodies (dilution, 1:1,000; cat. nos. sc-398303, sc-374388, sc-365241 and sc-376815; Santa Cruz Biotechnology, Inc., CA, USA); infrared-labeled fluores- 
cent goat anti-rabbit secondary polyclonal antibody (dilution, 1:2,000; cat. no. sc-2007; Santa Cruz Biotechnology, Inc.); western blotting kit (Shanghai Kangcheng Biotech Co., Ltd., Shanghai, China). All reagents were analytically pure and made in China.

The main instruments. Super clean bench (Suzhou Changqiao Purification Equipment Factory, Suzhou, China); Synergy 2 multifunctional microplate reader (BioTek Instruments, Winooski, VT, USA); ultraviolet spectrophotometer (Third Shanghai Analytical Instrument Factory, Shanghai, China); electronic scales (Sanyo, Tokyo, Japan); electrophoresis apparatus (Liuyi, Beijing, China); RT-PCR instrument (Shanghai Saimo Biological Science and Technology Development Co. Ltd., Shanghai, China).

Establishment of animal model. Three different hole areas, inlet hole, oxygen-measuring hole and outlet hole, were set up in a self-made oxygen box. The neonatal rats in the experimental group were fed in the self-made oxygen box (feeding conditions: oxygen concentration $\geq 95 \%$, humidity, $50-70 \%$ and temperature, $22-25^{\circ} \mathrm{C}$ ). Water and fodder were added regularly every day and the padding was replaced. Rats in the control group were placed in the standard mouse cage and fed by indoor air. The breathing, growth, development and activity of neonatal rats in both groups were monitored daily.

\section{Detection indexes and methods}

Specimen collection. At 14 days, rats were sacrificed using intraperitoneal injection of $10 \%$ chloral hydrate and the right lung was removed. Pieces of lung tissues were cryopreserved using liquid nitrogen and preserved at $-80^{\circ} \mathrm{C}$. The remaining lung tissues were fixed with $4 \%$ paraformaldehyde overnight for immunohistochemical and hematoxylin and eosin $(\mathrm{H} \& \mathrm{E})$ staining. The study was approved by the Ethics Committee of Children's Hospital of Zhengzhou and informed consents were signed by the patients and/or guardians.

Pulmonary pathological examination. Lung tissues were fixed with $4 \%$ paraformaldehyde overnight and were embedded in paraffin and cut into slices. After H\&E staining, radial alveolar count (RAC) and morphological changes in lung tissues were observed under microscope. The thickness of respiratory membrane was also detected using IPP6 image.

CASZI, Rho kinase, RhoA and ET-A receptor $m R N A$ s expression levels in lung tissues. Rat kidneys were removed and the total RNA was extracted using TRIzol method. Target gene primer sequences used were: GADPH (305 bp) forward, 5'-TGG TGAAGGTCGGTGTGAAC-3' and reverse primer, 5'-GGTGG TGAAGACGCCAGTAG-3'; CASZI (245 bp) forward, 5'-GGC ACATTCCGGGACAGAGAGAA-3' and reverse primer, 5'-CGG TTTACCCATGTGGTGCCTC-3'; RhoA forward,5'-ACCAGTT CCCAGAGGTGTATG-3' and reverse primer, 5'-TTGGGACAG AAGTGCTTGACTTC-3'; Rho kinase forward, 5'-CTGCGGGT ACGAAGGTATCG-3' and reverse primer, 5'-AGCATCCAAT CCATCCAGCA-3'; ET-A receptor forward, 5'-CCGAGGAGCT CTAAGGGGAA-3' and reverse primer, 5'-CCAAAAGGACG CCAGAAAGC-3'. The mRNA transcriptional levels of CASZI, Rho kinase, RhoA and ET-A receptor were detected via RT-PCR, and glyceraldehyde 3-phosphate dehydrogenase (GADPH) was used as internal reference.
CASZI, Rho kinase, RhoA and ET-A receptor proteins expression levels in lung tissues. Rat kidneys were removed and cut up using scissors. The total protein was extracted from the kidney tissues using pre-cooled tissue lysis buffer. The protein content in specimens was determined using Bradford method. The protein was separated on a $12 \%$ gel, and proteins were transferred onto the PVDF membrane using the membrane transfer instrument under $100 \mathrm{~V}$ for $1.5 \mathrm{~h}$. Membrane was sealed using skim milk powder for $2 \mathrm{~h}$. Membrane was washed, and consequently diluted monoclonal antibodies $(1: 1,000)$ anti-CASZI, Rho kinase, RhoA and ET-A receptor were added. After overnight incubation, membrane was washed, and the diluted secondary antibody was added followed by incubation at room temperature for $60 \mathrm{~min}$. BeyoECL Plus was used for color development, color-developing bands were collected using gel-imaging and chemiluminescence analysis system. Quantity One software was used for data analysis of protein bands.

Statistical analysis. All data were analyzed and treated using SPSS 18.0 software (SPSS, Inc., Chicago, IL, USA). Measurement data were presented as mean \pm SD and t-test was performed. $\mathrm{P}<0.05$ is considered to indicate a statistically significant difference.

\section{Results}

Morphological changes in lung tissues. In neonatal rats in the control group, alveoli had a uniform size and clear structure, the alveolar cavity was smaller and the alveolar septum was thicker without any obvious pathological changes. In neonatal rats in the experimental group, alveoli had different sizes with disordered structures. The alveolar wall was thinner, and we observed an increased alveolar volume, reduced area and fusion, suggesting that BPD occurred in lung tissue structures (Fig. 1).

$R A C$ and thickness of respiratory membrane. RAC in the experimental group was significantly lower than that in the control group $(\mathrm{P}<0.05)$ and the thickness of respiratory membrane was significantly higher in the experimental group $(\mathrm{P}<0.05)$ (Table I).

CASZI, Rho kinase, RhoA and ET-A receptor $m R N A$ expression levels in lung tissues. CASZI mRNA level in the experimental group was significantly lower than that in the control group $(\mathrm{P}<0.05)$, and Rho kinase, RhoA and ET-A receptor mRNA levels were significantly higher than those in the control group $(\mathrm{P}<0.05)$ (Fig. 2).

CASZI, Rho kinase, RhoA and ET-A receptor protein expression levels in lung tissues. CASZI protein level in the experimental group was significantly lower than that in the control group $(\mathrm{P}<0.05)$, while Rho kinase, RhoA and ET-A receptor proteins levels were significantly higher in the experimental group $(\mathrm{P}<0.05)$ (Fig. 3).

\section{Discussion}

The complex pathogenesis of BPD is a multi-factor process involving the interaction among epithelial cells, pulmonary 

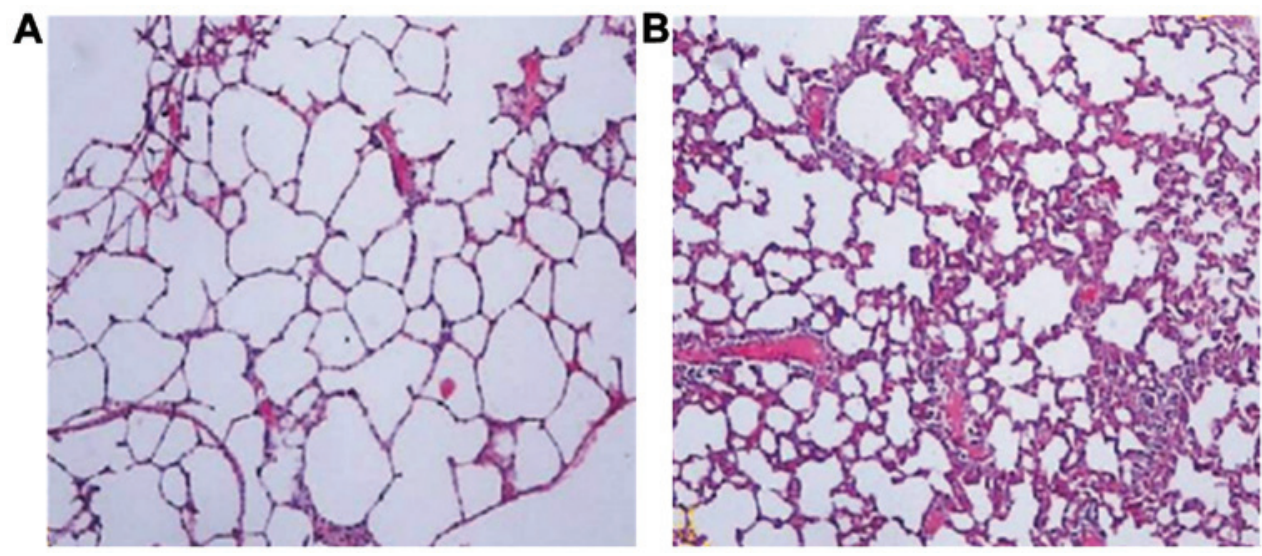

Figure 1. Morphological changes in lung tissues. (A) control group, (B) experimental group.

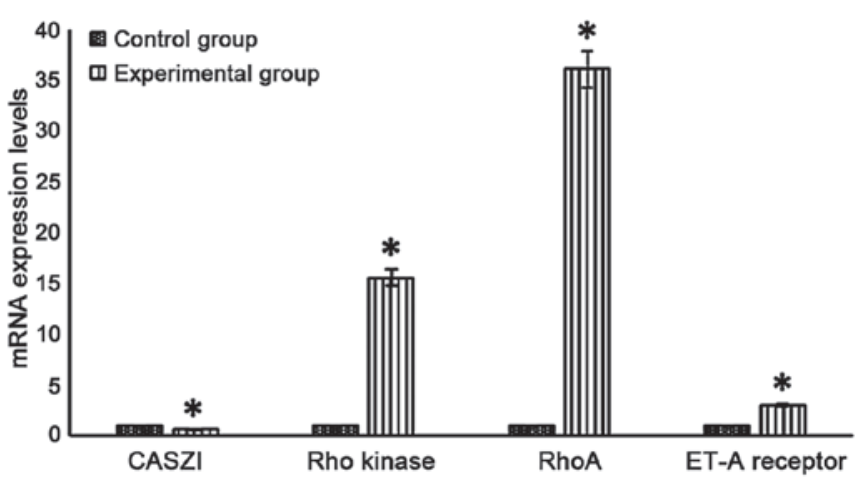

Figure 2. CASZI, Rho kinase, RhoA and ET-A receptor mRNA expression levels in neonatal rats in the experimental group and the control group $\left({ }^{*} \mathrm{P}<0.05\right.$ vs. control group).

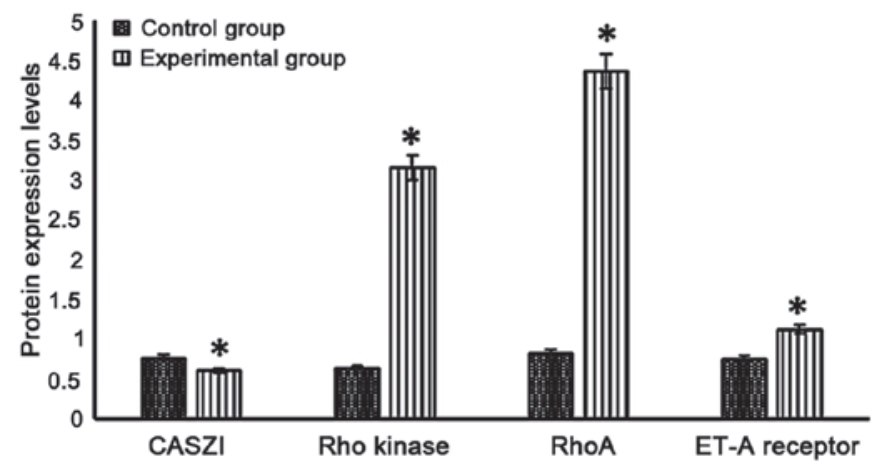

Figure 3. CASZI, Rho kinase, RhoA and ET-A receptor protein expression levels in neonatal rats in the experimental group and the control group $\left({ }^{*} \mathrm{P}<0.05\right.$ vs. control group).

interstitium and pulmonary microvascular system (6). The alveolarization damage and pulmonary microvascular abnormalities are the two important poles in the pathogenesis of BPD (7). In recent years, more attention has been focused on the importance of the pulmonary microvascular abnormalities in BPD, and to emphasize this importance, some scholars have introduced the 'vascular hypothesis' (8).

In this study, BPD rat model was established using 95\% hyperoxia. The lung development in neonatal rats was in the vesicular stage, equivalent to human gestational age
Table I. RAC and thickness of respiratory membrane of neonatal rats in experimental group and control group.

\begin{tabular}{lcc}
\hline Group & RAC (n) & $\begin{array}{c}\text { Thickness of respiratory } \\
\text { membrane }(\mu \mathrm{m})\end{array}$ \\
\hline Experimental group & $5.6 \pm 0.5$ & $11.9 \pm 2.3$ \\
Control group & $8.2 \pm 0.9$ & $6.9 \pm 1.5$ \\
t-value & 2.308 & 2.275 \\
P-value & 0.015 & 0.022 \\
\hline
\end{tabular}

RAC, radial alveolar count.

of $\sim 28$ weeks. At this time, the lung development was not perfect with active shaping and remodeling. Hyperoxia method induced was similar to the occurrence and development process of BPD in premature infants, and with the ability of damaging and inhibiting the alveoli and can cause acute lung injury in the short term. The experimental data showed that in neonatal rats in the control group, alveoli had a uniform size with clear structure. The alveolar cavity was smaller and the alveolar septum was thicker without obviously pathological changes. In the experimental group, alveoli had various sizes with disordered structures. The alveolar wall was thinner, and an increased alveolar volume was observed. In the experimental group, we observed lower area and fusion, suggesting that BPD occurred in lung tissue structures. RAC in the experimental group was significantly lower than that in the control group $(\mathrm{P}<0.05)$ and the thickness of respiratory membrane was significantly higher than that in the control group $(\mathrm{P}<0.05)$. These observations showed that $\mathrm{BPD}$ rat model was successfully established using $95 \%$ hyperoxia.

Vasculogenesis and angiogenesis are two major processes of vascular generation, the former is observed when the new blood vessels are formed by endothelial cells, endothelial progenitor cells or hemangioblastoma from nothing, and the latter is observed when the new blood vessels are formed based on the existing blood vessels through promoting the vascular endothelial cell migration and proliferation $(9,10)$. Pulmonary angiogenesis involves a complex regulatory factors network, and several pro-vascular growth factors have been 
already discovered. These factors include angiopoietin (Ang), vascular endothelial growth factor (VEGF), placental growth factor (PlGF), tyrosine kinase receptor-ligand (EGF/Ephrin), platelet-derived growth factor (PDGF), basic fibroblast growth factor (bFGF), transforming growth factor- $\beta$ (TGF- $\beta$ ), hepatocyte growth factor (HGF) and monocyte chemoattractant protein-1 (11-13). CASZI is a recently-discovered transcription factor which belongs to the zinc finger gene family. CASZI is widely distributed in the vascular endothelial cells in lung, stomach, pancreas, testis, skeletal muscle, heart and small intestine. CASZI binds to DNA and plays an important role in cell proliferation, differentiation and apoptosis. It has been proved that CASZI can promote the formation and branching of vascular networks, as well as promoting vascular buds (14). The results of this study showed that CASZI mRNA and protein expression levels in the experimental group were significantly lower than those in the control group $(\mathrm{P}<0.05)$. These results suggested that high-concentration of oxygen could inhibit the expression of CASZI, and CASZI may play an important role in the development of BPD.

In order to further study the regulatory mechanism of lung development, Rho kinase, RhoA and ET-A receptor mRNA and protein expression levels in RhoA/ROCK were analyzed. RhoA/ROCK is a downstream signaling pathway of CASZI, and RhoA is an important cellular signal transduction molecule, which is essentially a GTPase $(15,16)$, and its main role is to hydrolyze ornithine. Activated RhoA has the capability of influencing a variety of biological behaviors, such as cell cycle regulation, transcription factor activation and smooth muscle contraction (16). Rho kinase is a downstream signaling protein of RhoA, and G-protein-coupled receptor can activate RhoA through ET receptor, thus activating Rho kinase and mediating the differentiation, proliferation and migration of vascular endothelial cells $(17,18)$. Prior studies have confirmed that CASZI can regulate the endothelial cell adhesion through RhoA, and promote vascular buds. The experimental data showed that Rho kinase, RhoA and ET-A receptor protein expression levels in the experimental group were significantly higher than those in the control group $(\mathrm{P}<0.05)$. Therefore, it is speculated that hyperoxia may inhibit CASZI expression, thus downregulating the expression of RhoA, activating RhoA/ROCK pathway and reducing the formation and branching of pulmonary vascular network.

We concluded that the expression of CASZI, during the development of BPD, was downregulated, and this influenced the pulmonary microvascular regeneration through RhoA/ROCK signaling pathway, thus participated in the pathogenesis of BPD.

\section{References}

1. Gien J, Kinsella J, Thrasher J, Grenolds A, Abman SH and Baker CD: Retrospective analysis of an interdisciplinary ventilator care program intervention on survival of infants with ventilatordependent bronchopulmonary dysplasia. Am J Perinatol 34: 155-163, 2017.

2. Nair V, Loganathan P and Soraisham AS: Azithromycin andother macrolides for prevention of bronchopulmonarydysplasia: A systematic review and meta-analysis. Neonatology 106: 337-347, 2014.
3. Gadhia MM, Cutter GR, Abman SH and Kinsella JP: Effects of early inhaled nitric oxide therapy and vitamin A supplementation on the risk for bronchopulmonary dysplasia in premature newborns with respiratory failure. J Pediatr 164: 744-748, 2014.

4. Hu Q, Liu X and Zhou G: Effects of ligustrazine on hypoxiainducible factor pathway expression in neonatal rats with BPD. J Pediatr Pharm 20: 1-5, 2014.

5. Golovnin AK, Kostyuchenko MV, Georgiev PG and Melnikova LS: Mod(mdg4)-58.8, isoform of $\bmod (m d g 4)$ loci, directly interacts with MTACP1A and MTACP1B proteins of Drosophila melanogaster. Dokl Biochem Biophys 466: 5-8, 2016.

6. Vom Hove M,Prenzel F, Uhlig HH and Robel-Tillig E: Pulmonary outcome in former preterm, very low birth weight children with bronchopulmonary dysplasia: A case-control follow-up at school age. J Pediatr 164: 40-45.e4, 2014

7. Balany $\mathrm{J}$ and Bhandari V: Understanding the impact of infection, inflammation, and their persistence in the pathogenesis of bronchopulmonary dysplasia. Front Med (Lausanne) 2: 90, 2015.

8. Del Re DP, Miyamoto S and Brown JH: RhoA/Rho kinase upregulate Bax to activate a mitochondrial death pathway and induce cardiomyocyte apoptosis. J Biol Chem 282: 8069-8078, 2007.

9. Monteiro CB, Midão L, Rebelo S, Reguenga C, Lima D and Monteiro FA: Zinc finger transcription factor Casz1 expression is regulated by homeodomain transcription factor Prrxl1 in embryonic spinal dorsal horn late-born excitatory interneurons. Eur J Neurosci 43: 1449-1459, 2016.

10. Wang L, Feng $Z$ and Lv H: Expressions of vascular endothelial growth factor and angiopoietin-1 in neonatal rats with hypoxiainduced BPD and effects on lung development. J Pract Med 20: 525-528, 2014.

11. Tang H: 'Vascular hypothesi' of BPD and progress of Angiogenesis-promoting Treatment. J Clin Pediatr 34: 553, 2016.

12. Liu Z, Lam N and Thiele CJ: Zinc finger transcription factor CASZ1 interacts with histones, DNA repair proteins and recruits NuRD complex to regulate gene transcription. Oncotarget 6: 27628-27640, 2015.

13. Li Q, Ma X and Zhang X: Pulmonary microvascular development and CD34 expression in rats with oxygen-induced BPD. J PLA Med 26: 25-28, 2014.

14. Sauzeau V, Rolli-Derkinderen M, Lehoux S, Loirand G and Pacaud P: Sildenafil prevents change in RhoA expression induced by chronic hypoxia in rat pulmonary artery. Circ Res 93: 630-637, 2003

15. Pierro M, Ionescu L, Montemurro T, Vadivel A, Weissmann G, Oudit G, Emery D, Bodiga S, Eaton F, Péault B, et al: Short-term, long-term and paracrine effect of human umbilical cord-derived stem cells in lung injury prevention and repair in experimental bronchopulmonary dysplasia. Thorax 68: 475-484, 2013.

16. Julian L and Olson MF: Rho-associated coiled-coil containing kinases (ROCK): Structure, regulation, and functions. Small GTPases 5: e29846, 2014.

17. Stiles JM, Kurisetty V, Mitchell DC and Bryan BA: Rho kinase proteins regulate global miRNA expression in endothelial cells. Cancer Genomics Proteomics 10: 251-263, 2013.

18. Kaya G, Sivasli E, Oztuzcu S, Melekoglu NA, Ozkara E, Sarikabadayi U and Demiryürek AT: Association of Rho-kinase gene polymorphisms with respiratory distress syndrome in preterm neonates. Pediatr Neonatol 58: 36-42, 2017. 